\title{
Efeito Payne em Nanocompósitos de NBR com Montmorilonita Organofílica
}

\author{
Fernando de $\mathbf{0}$. Bezerra \\ Instituto Federal de Educação, Ciência e Tecnologia do Rio de Janeiro, Campus Realengo \\ Regina C. R. Nunes, Ailton S. Gomes \\ Instituto de Macromoléculas Professora Eloísa Mano \\ Márcia G. Oliveira \\ Instituto Nacional de Tecnologia \\ Edson N. Ito \\ Universidade Federal do Rio Grande do Norte
}

\begin{abstract}
Resumo: Neste trabalho foi estudado o efeito Payne como uma medida das interações carga-carga e polímero-carga em nanocompósitos de montmorilonitaorganofílica (MMTorg) e borracha nitrílica (NBR), usando o Analisador de Processamento de Borracha - RPA 2000. Os nanocompósitos de NBR e MMTorg foram preparados em um misturador de rolos e o efeito Payne foi avaliado em compostos não vulcanizados tipo goma pura e contendo 5, 10, 15 e 20 phr de MMTorg. Os resultados mostraram que a composição com 5phr de MMTorg apresentou melhor interação polímero-carga como consequência da menor quantidade de aglomerados de carga na matriz elastomérica.
\end{abstract}

Palavras-chave: Nanocompósito, montmorilonita organofílica, efeito Payne, borracha nitrílica, RPA 2000.

\section{Payne Effect in NBR Nanocomposites with Organofilic Montmorillonite}

Abstract: In this work the Payne effect was evaluated as a measure of the filler-filler and filler-polymer interactions in nanocomposites of organomontmorillonite (MMTorg) on acrylonitrile-butadiene-rubber (NBR) by using the Rubber Process Analyzer - RPA 2000. The nanocomposites of NBR and MMTorg were prepared on a Berstorff two-roll mill and the evaluation of the Payne effect was carried out in unvulcanized pure gum and varying the MMTorg content from 5, 10, 15 and 20 phr. The composition with 5 phr of MMTorg showed the best filler-polymer interaction as a consequence of the smaller amount of agglomerates of the filler in the elastomeric matrix.

Keywords: Nanocomposite, organofilic montmorillonite, Payne effect, acrylonitrile-butadiene-rubber, RPA 2000.

\section{Introdução}

Nanocompósitos poliméricos compreendem uma nova classe de materiais híbridos onde substâncias inorgânicas com pelo menos 100 nanômetros em uma das dimensões encontram-se dispersas em uma matriz polimérica ${ }^{[1-11]}$.

Estruturas em escala nanométrica, como argilominerais, possuem elevados níveis de superfície específica, podendo atingir $800 \mathrm{~m}^{2} / \mathrm{g}$ e alta razão de aspecto (L/D - comprimento/espessura) de aproximadamente 1000:1, tornando-se ideais para uso em materiais poliméricos ${ }^{[2,4,7,10,11]}$.

Os nanocompósitos obtidos com argilas organofílicas têm atraído a atenção da classe científica, pela melhoria nas propriedades mecânicas, térmicas, de barreira, estabilidade dimensional e propriedades retardantes de chama inferidos aos polímeros, sendo isso possível com teores baixos de nanoargilas, geralmente da ordem de 2 a $10 \mathrm{phr}$, acarretando inclusive redução da densidade ${ }^{[3,4,6-13]}$.

Com o advento da tecnologia de nanocompósitos poliméricos utilizando argilas organofílicas, em especial a montmorilonita, tornou-se importante estudar a função dessas nanocargas na matriz elastomérica, para melhor compreender o reforço causado por este tipo de argilomineral. O reforço está relacionado com as propriedades básicas da carga, como área específica superficial (tamanho de partículas), estrutura (formação de agregados de carga) e atividade superficial das partículas (interações físicas e químicas), teor, modo de processamento e morfologia final do compósito em relação a sua dispersão e distribuição na matriz polimérica $^{[3,14-21]}$.

A adição de carga em elastômeros modifica as propriedades físicas dos polímeros elastoméricos afetando significativamente as propriedades viscoelásticas dinâmicas dos artefatos de borracha $^{[10,14-24]}$. O comportamento viscoelástico dinâmico de elastômeros com carga em função da amplitude de deformação é conhecido como Efeito Payne, e está ilustrado na Figura $1^{[25-33]}$.

Payne descobriu que a estrutura da rede tridimensional construída pela agregação de partículas de cargas, como o negro de fumo, com comportamento similar para sílica e argilas, é um dos fatores responsáveis pelo desempenho dinâmico de artefatos elastoméricos. A estrutura em rede formada pela carga (interação carga-carga) é um fator dominante no aumento da histerese, e está diretamente relacionada a quebra e reconstituição dessas estruturas de agregados secundários em compostos de borrachas com cargas ${ }^{[30-31]}$.

Segundo o modelo de Payne (Figura 1) o reforço é o somatório das contribuições do efeito da rede de borracha sem carga, do efeito hidrodinâmico da carga, das interações borracha-carga e carga-carga ${ }^{[26,28,31,32]}$. A contribuição da rede da borracha sem carga depende da natureza estrutural do polímero bem como da densidade de ligações cruzadas ${ }^{[26,28,31,32]}$. O efeito hidrodinâmico está relacionado com a grandeza da deformação, resultante da diluição das partículas da carga na borracha, considerando que a

Autor para correspondência: Regina C. R. Nunes, Instituto de Macromoléculas Professora Eloisa Mano - IMA, Universidade Federal do Rio de Janeiro - UFRJ, CEP 21945-970, Rio de Janeiro, RJ, Brasil, e-mail: rcnunes@ima.ufrj.br 
carga é a fase rígida que não pode ser deformada ${ }^{[26,28,31,32]}$. O efeito da interação da carga - polímero é atribuído a morfologia da carga no polímero e a natureza química da matriz. A estrutura da carga no polímero é a quantidade de borracha ocluida entre os espaços vazios de um agregado de carga e esta interação acarreta um aumento do módulo (resistência mecânica). A interação borracha-carga pode ser atribuída a ligações físicas (Van der Waals-London), bem como químicas ou uma mistura de ambas ${ }^{[26,28,31,32]}$.

O efeito Payne pode ser caracterizado pelo módulo elástico a baixas deformações como resultante das ligações carga-carga dos interagregados. A medida que a amplitude de deformação aumenta, a rede de ligações carga-carga dos interagregados é quebrada. A diferença entre os valores de módulo de cisalhamento elástico a baixa e alta deformação $\left(\mathrm{G}_{0}{ }_{0}-\mathrm{G}^{\prime} \infty\right)$ permite identificar a influência de uma carga específica em uma determinada matriz elastomérica ${ }^{[28-32]}$.

As propriedades viscoelásticas são importantes na indústria de borracha, pois permitem uma avaliação do desempenho do artefato final em aplicações dinâmicas. Um exemplo bem típico é na indústria de pneumáticos, visando alcançar resistência a derrapagem com menor consumo de energia, de forma a atender a legislação ambiental, tecnologia esta conhecida como "Green Tyre". Estas propriedades estão relacionadas, por exemplo, com a variação do módulo em função da frequência ou da deformação, como é o caso do efeito Payne ${ }^{[34,35]}$.

Um dos equipamentos que vêm sendo usado com sucesso na determinação das propriedades dinâmicas de elastômeros é o Analisador de Processamento de Borracha (RPA 2000). Esse equipamento permite o levantamento das propriedades viscoelásticas de elastômeros puros e composições, antes, durante e após a cura, de uma forma rápida e simples. As variáveis do equipamento são: deformação, temperatura, tempo e frequência ${ }^{[36-41]}$.

A diferença $\Delta \mathrm{G}$ 'entre o módulo elástico em baixas deformações $\left(G_{0}^{\prime}\right)$ e em altas deformações $\left(G^{\prime} \infty\right)$ é uma quantificação do efeito Payne, atribuída à estrutura da carga e pode ser entendida como ligações carga-carga de natureza física dos interagregados (forças de van der Waals-London) que são quebradas em altas deformações ${ }^{[28-32]}$.

Neste trabalho foi possível utilizar o conceito de efeito Payne para avaliar as interações carga-carga e carga-polímero em nanocompósitos de borracha nitrílica (NBR) com montmorilonita organofílica (MMTorg) usando o Analisador de Processamento de Borracha - RPA 2000.

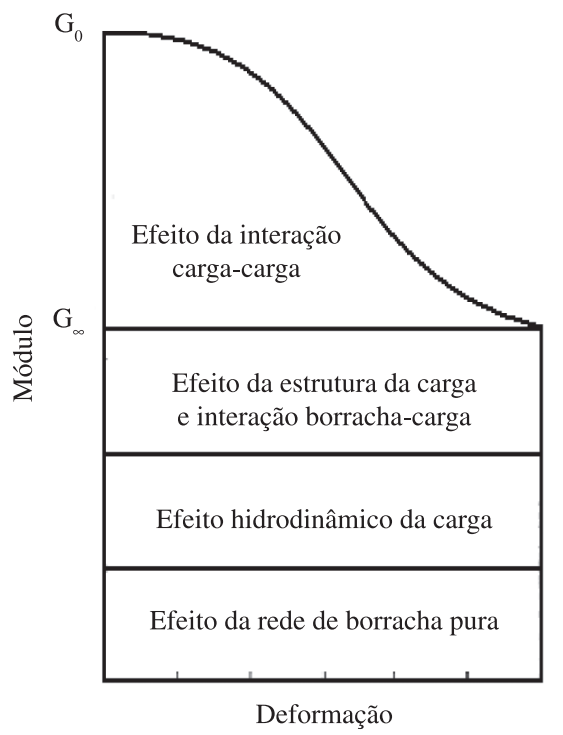

Figura 1. Efeito das contribuições no reforço segundo Payne $e^{[26,28,31,32]}$.

\section{Experimental}

\section{Materiais}

Os materiais usados neste trabalho foram: a) Argila organofílica VISCOGEL B7 da BENTEC S.p.A. (Itália), cujas características informadas pelo fabricante estão apresentadas na Tabela 1; b) Borracha nitrílica N615B da Nitriflex S.A. Indústria e Comércio (Brasil), (Tabela 2); c) Ácido esteárico duplamente granulado, da Cia Estearina Paranaense Ltda, pureza 99\% (Brasil); d) Óxido de Zinco, da Unyroyal Química S.A., grau de pureza P.A (Brasil); e) TBBS (t-butil-2-benzotiazol-sulfenamida), da Flexys Indústria e Comércio Ltda (Brasil); f) Enxofre rômbico ou monoclínico $\left(\mathrm{S}_{8}\right)$, da Stauffer Chemical Company (USA), pureza 97-100\%.

\section{Formulação, preparo dos corpos de prova e processamento dos nanocompósitos}

Composições de NBR tipo goma pura, e com 5, 10, 15 e 20 phr de MMTorg foram preparadas em misturador de rolos da marca Berstorff, a $50{ }^{\circ} \mathrm{C}$, em duas etapas. Na primeira etapa foram obtidos os masterbatches de NBR/MMTorg durante uma hora de mistura, com razão de fricção no misturador de rolos de 1:1,50, abertura entre os rolos de $1,40 \mathrm{~mm}(0,055 \mathrm{in})$ e temperatura de $50^{\circ} \mathrm{C}$. Na segunda etapa procedeu-se a incorporação do sistema de cura (ativadores e aceleradores) por 25 minutos, na temperatura de $50{ }^{\circ} \mathrm{C}$, de acordo com a norma ASTM D 3187-90, e com razão de fricção entre rolos de 1:1,25. A formulação usada foi a seguinte, (em phr): NBR 100; óxido de zinco 3; ácido esteárico 1,0; enxofre 1,5; TBBS (terc-butil benzotiazol sulfenamida) 0,7; e MMTorg (0, 5, 10, 15, 20).

O efeito Payne dos nanocompósitos de NBR/MMTorg foi obtido em composições não-curadas, a partir da determinação do módulo elástico (G') nas seguintes condições experimentais: temperatura, $100{ }^{\circ} \mathrm{C}$; frequência, $1 \mathrm{~Hz}(60 \mathrm{cpm})$; deformação em ângulos de 0,5 a $30^{\circ}(7 \%$ a $420 \%)$. O equipamento usado foi o Analisador

Tabela 1. Características físico-químicas da montmorilonita organofílica (MMTorg).

\begin{tabular}{ll}
\hline \multicolumn{1}{c}{ Propriedades } & \multicolumn{1}{c}{ Resultados } \\
\hline Composição & Argila esmectita com sal \\
& quaternário de alquilamônio \\
Fórmula estrutural do sal & $R$ \\
quaternário & $R: \ddot{N}: R+X^{-}$ \\
de amônio & $R$ \\
( $\mathrm{R}=$ radical orgânico, & \\
$\mathrm{X}=$ Halogênio $)$ & Bege clara \\
Cor & pó \\
Forma & 1,7 \\
Densidade $\left(\mathrm{g} / \mathrm{cm}^{3}\right)$ & 3,0 \\
Umidade $(\%)$ & 18,0 \\
Área específica $\left(\mathrm{m}^{2} / \mathrm{g}\right)$ & 17,76 \\
Distância interplanar $\left(\mathrm{d}_{001}\right)(\AA)$ & De média a alta polaridade \\
Polaridade/uso & \\
\hline
\end{tabular}

Tabela 2. Propriedades da borracha nitrílica N-615B.

\begin{tabular}{lcc}
\hline \multicolumn{1}{c}{ Propriedades } & Resultado & Especificação \\
\hline Aparência & $\begin{array}{c}\text { Bege clara a } \\
\text { amarelada }\end{array}$ & $\begin{array}{c}\text { Bege clara a } \\
\text { amarelada }\end{array}$ \\
Acrilonitrila combinada $(\%)$ & 31,0 & $31,0-34,0$ \\
Teor de cinzas $(\%)$ & 0,30 & $0,00-1,00$ \\
Viscosidade Mooney & 52 & $40-54$ \\
$\left(\right.$ MML 1+4 @ $\left.100^{\circ} \mathrm{C}\right)$ & & \\
\hline
\end{tabular}


de Processamento de Borracha (Rubber Process Analyser - RPA 2000) desenvolvido pela Monsanto Instruments, fabricado e comercializado pela Alpha Technologies.

\section{Preparação dos corpos de prova para análise de DRX e TEM}

Os corpos de prova para análise de DRX e TEM foram obtidos por compressão, a $150{ }^{\circ} \mathrm{C}$, aplicando uma pressão de aproximadamente 4,4 MPa, no tempo ótimo de vulcanização $\left(\mathrm{t}_{90}\right)$ de cada composição, obtido pelo Reômetro de Disco Oscilatório - ODR em arco de $1^{\circ}$, que foi de 30 minutos.

\section{Difração de raios-X}

A difração de raios-X (XRD) é uma técnica utilizada no estudo da estrutura dos polímeros em geral e dos minerais em particular. Esta técnica é uma ferramenta poderosa e simples na avaliação de materiais contendo arranjos periódicos de elementos, como a estrutura dos silicatos em nanocompósitos intercalados, mas fornece pouca informação em estruturas de silicatos esfoliados, pois estes não possuem arranjo periódico ${ }^{[1-15,42,43]}$.

Neste trabalho as composições de NBR tipo goma pura e contendo 5, 10, 15 e 20 phr de MMTorg foram analisadas no Difratômetro de raios-X - tipo Miniflex Rigaku Corporation - Japão.

$\mathrm{O}$ espaçamento interlamelar pode ser calculado utilizando a lei de Bragg: $n \lambda=2$ d sen $\theta$, onde " $n$ " é igual a 1 , " $\lambda$ " corresponde ao comprimento de onda da radiação de raios-X utilizada $(\lambda=1,51418 \AA)$, "d" o espaçamento entre as lamelas da argila e " $\theta$ " a medida do ângulo de difração. A intercalação das cadeias poliméricas entre as lamelas da argila aumenta esse espaçamento, em comparação ao espaçamento interlamelar da argila original, levando à mudanças do pico de difração no sentido de valores de baixos ângulos ${ }^{[2,42]}$.

\section{Microscopia eletrônica de transmissão - TEM}

A microscopia eletrônica de transmissão é uma ferramenta importante para monitorar, por visualização direta, a presença de nanomateriais, sua dispersão e distribuição em uma matriz ${ }^{[1-11,14-17]}$. Esta técnica foi usada neste trabalho para a confirmação dos nanocompósitos obtidos pelo processamento adotado. Os cortes das amostras foram realizados em um ultramicrótomo da marca RM modelo MT 7000, usando uma faca de diamante da marca Diatome tipo CryoHisto $45^{\circ}$, com a temperatura de corte de $-80^{\circ} \mathrm{C}$ resfriada com nitrogênio líquido, para evitar a deformação da microestrutura original, com velocidade de corte de $0,1 \mathrm{~mm} / \mathrm{s}$ e espessura de corte de $25 \mathrm{~nm}$. As amostras crio-ultramicrotomadas foram analisadas em um microscópio eletrônico de transmissão, equipamento da Philips modelo CM120, com tensão de $120 \mathrm{kV}$.

\section{Analisador de Processamento de Borracha - RPA 2000}

O Analisador de Processamento de Borracha - RPA 2000 é um aparelho de teste reológico mecânico dinâmico (DMRT) projetado para medir propriedades de borrachas puras, em composições não curadas e curadas. O RPA 2000 aplica tensão de cisalhamento em uma amostra através de oscilação do bloco inferior senoidalmente, em uma cavidade selada e pressurizada (Figura 2). A frequência de oscilação pode ser ajustada de 0,0016 a $50 \mathrm{~Hz}$ (ou 0,1 a 3000 ciclos por minutos - cpm) em uma ampla faixa de ângulos de deformação, como de $\pm 0,02$ até $\pm 90,00$ graus (correspondendo a $\pm 0,3 \%$ até $\pm 1250 \%$ ) e a temperatura pode ser variada de ambiente até $230{ }^{\circ} \mathrm{C}^{[36-41]}$.

O equipamento RPA 2000 foi utilizado na determinação do efeito Payne dos nanocompósitos de NBR/MMTorg, a partir da determinação do módulo elástico (G') para cada composição em função da deformação de 7 a $420 \%$ (ângulo de 0,5 a $30^{\circ}$ ), em

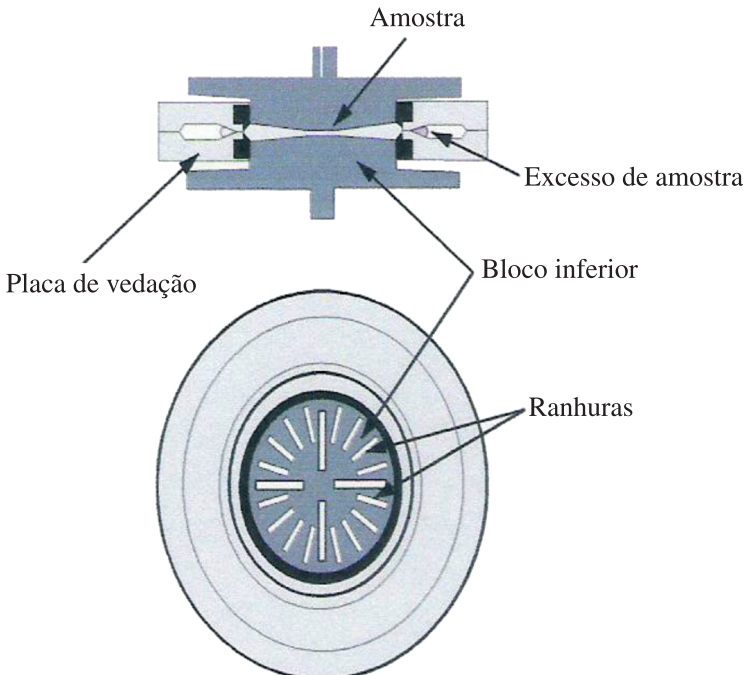

Figura 2. Câmara de teste do RPA 2000 ${ }^{[39]}$

composições não vulcanizadas de NBR com MMTorg, fixando a temperatura em $100{ }^{\circ} \mathrm{C}$ e frequência de $1 \mathrm{~Hz}(60 \mathrm{cpm})$.

\section{Resultados e Discussão}

\section{Caracterização dos nanocompósitos de NBR com montmorilonita organofilica}

Técnicas como difração de raios-X (XRD) e microscopia eletrônica de transmissão (TEM) são importantes na caracterização de nanocompósitos com argila. Estas técnicas permitem avaliar o grau de dispersão/distribuição da montmorilonita organofílica na matriz elastomérica, que estão diretamente ligados ao reforço proporcionado $^{[1-17,42]}$.

No caso de XRD, o deslocamento dos picos difratados a ângulos $2 \theta$ menores significa aumento da distância interplanar $\left(\mathrm{d}_{001}\right)$ da argila, pela presença de moléculas de polímeros entre suas lamelas, fenômeno conhecido como intercalação. Quando o aumento da distância interplanar for maior do que as forças de interação entre as lamelas da argila (forças de van der Waals-London), ocorre o rompimento destas interações, apresentando estruturas esfoliadas, significando ausência de picos por difração no XRD ${ }^{[1-6,10,14,41]}$.

Na Figura 3, é possível observar os picos de difração dos nanocompósitos obtidos, e comprovar a formação de estrutura tipo esfoliada com $5 \mathrm{phr}$ de carga, e estruturas intercaladas e/ou esfoliadas nas demais amostras. Além disso, com o aumento da carga, ocorrem picos em torno de $2 \theta=6,5^{\circ}$. O deslocamento dos picos para valores de ângulos maiores é ocasionado pela diminuição da distância interplanar da argila. Portanto, uma das causas para o aparecimento destes picos pode estar relacionada com o polímero envolvendo os aglomerados de argila. Outra justificativa pode estar relacionada com a reagregação das lamelas de argila.

A Figura 4 mostra a fotomicrografia por TEM do nanocompósito de NBR com 10 phr de montmorilonita organifílica (MMTorg). É possível observar, em escala nanométrica, a dispersão/distribuição de MMTorg na matriz elastomérica, comprovando a obtenção de nanocompósitos, e corroborando os resultados de XDR.

\section{Efeito Payne}

As interações carga-matriz e carga-carga nas composições desenvolvidas foram avaliadas pelo efeito Payne 20-29,42 $^{2}$ através 


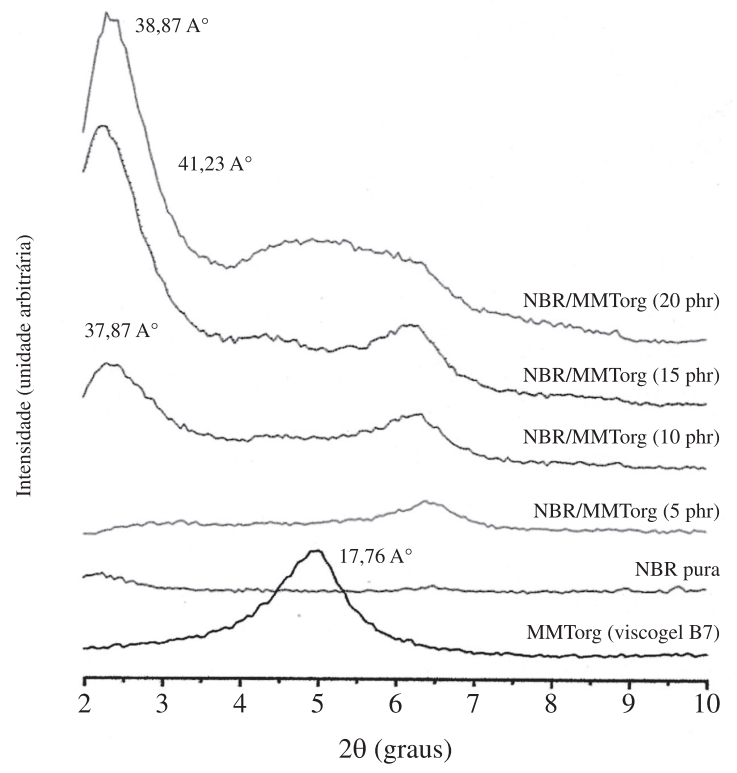

Figura 3. Difração de raios-X (XDR) dos nanocompósitos NBR/MMTorg.

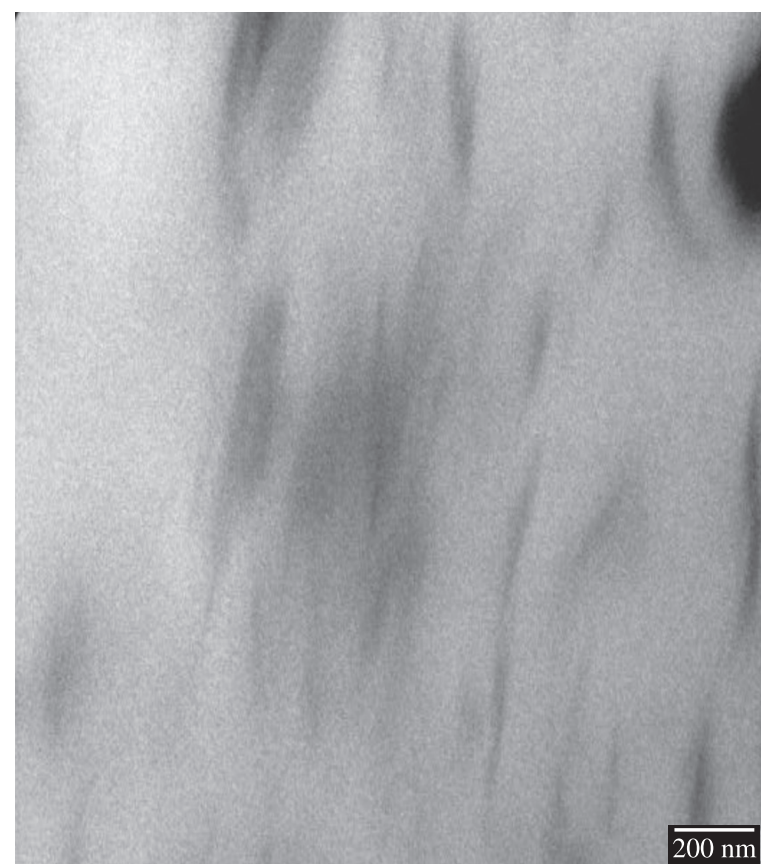

Figura 4. Fotomicrografia por TEM de NBR com 10 phr de MMTorg.

do estudo do módulo elástico em função de uma larga faixa de deformação, mantendo constantes a frequência e a temperatura. Os resultados são mostrados nas Figuras de 5 a 7.

Os resultados do presente trabalho, tendo MMTorg como carga são analisados em comparação ao estudo do efeito Payne com negro de fumo. A diferença entre $G_{0}^{\prime}$ e $G^{\prime} \infty$ (G'14\%-G'210\%), denominada $\Delta G^{\prime}$, é uma medida do efeito Payne atribuída a estrutura da carga, e pode ser visualizada como interações cargacarga de natureza física, que são quebradas em altas deformações e tem sido usada para quantificar as interações dos interagregados (ligações de van der Waals). O efeito Payne será maior quanto maior for $\Delta \mathrm{G}$, indicando uma maior quebra de interações carga-carga e, consequentemente, maior quantidade de aglomerados de carga na matriz elastomérica ${ }^{[25-33]}$.

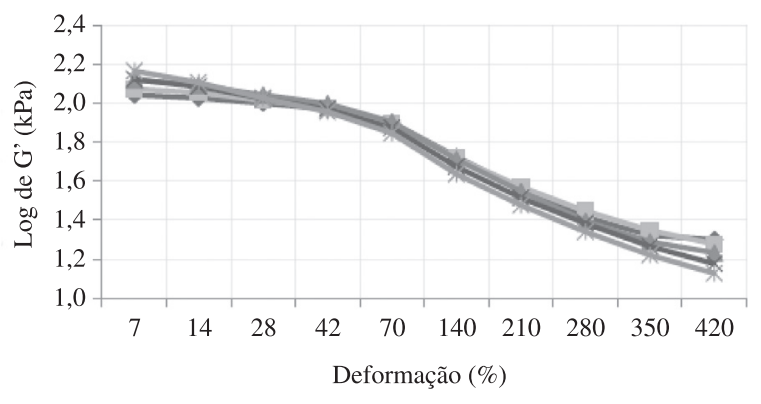

$\because 100 / 0 \div-100 / 5 \div 100 / 10 \div 100 / 15 \div 100 / 20$

Figura 5. Log de G' em função da deformação dos nanocompósitos NBR/ MMTorg. Condições experimentais: temperatura de $100{ }^{\circ} \mathrm{C}$ e frequência de $60 \mathrm{com}$.

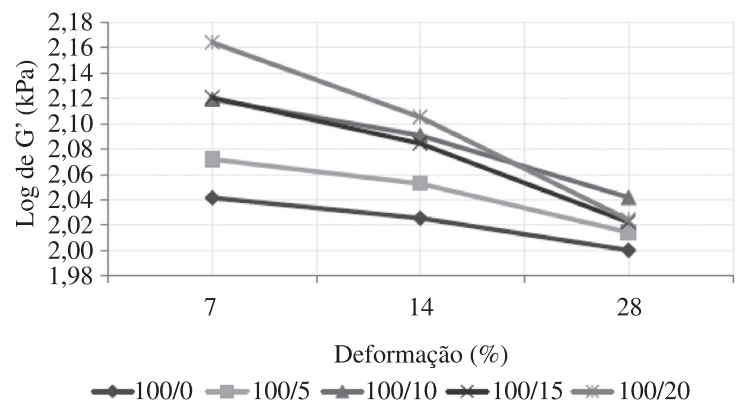

Figura 6. Log de G' a baixas deformações (7 a 28\%) de nanocompósitos NBR/MMTorg. Condições experimentais: temperatura de $100{ }^{\circ} \mathrm{C}$ e frequência de $60 \mathrm{cpm}$.

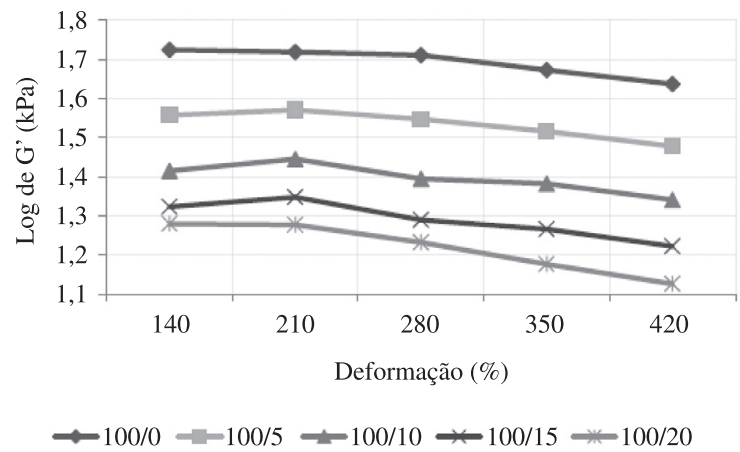

Figura 7. Log de G' a altas deformações (140 a 420\%) de nanocompósitos NBR/MMTorg. Condições experimentais: temperatura de $100{ }^{\circ} \mathrm{C}$ e frequência de $60 \mathrm{com}$.

Na Figura 5 estão mostrados os dados para toda a faixa de deformação estudada ( 0 a 420\%), e nas Figuras 6 e 7 os resultados estão mais bem definidos para a faixa de baixa deformação (0 a $28 \%$ ) e alta deformação (140 a $420 \%$ ), respectivamente.

Pelos resultados observa-se que a baixas deformações (Figura 6) os nanocompósitos de NBR/MMTorg apresentam o módulo elástico (G') crescente com o aumento do teor de MMTorg. Este aumento é causado pela formação das interações carga-carga, cuja distância entre os interagregados torna-se cada vez menor com o conteúdo da carga. Por outro lado, segundo a mesma teoria, em altas deformações (Figura 7), onde há o rompimento das interações dos interagregados, o efeito predominante é de interações polímero-carga.

Os resultados indicam a composição de 5 phr MMTorg como a de melhor desempenho mecânico como consequiência da menor quantidade de aglomerados de carga rompidos, e de melhor interação com a matriz de NBR, provavelmente de natureza química. 
Tabela 3. Efeito do teor de MMTorg no módulo dinamico em função da deformação (Efeito Payne) em nanocompósitos de NBR.

\begin{tabular}{cc}
\hline $\begin{array}{c}\text { Nanocompósitos } \\
\text { NBR/MMTorg }\end{array}$ & $\begin{array}{c}\text { Efeito Payne } \\
\left(\Delta \mathbf{G}^{\prime}=\mathbf{G}^{\prime} \mathbf{1 4} \%-\mathbf{G}^{\prime} \mathbf{2 1 0} \%\right)\end{array}$ \\
\hline $100 / 5$ & 76 \\
$100 / 10$ & 88 \\
$100 / 15$ & 89 \\
$100 / 20$ & 97 \\
\hline
\end{tabular}

Na Tabela 3 são mostrados os valores da diferença de módulo elástico a altas e baixas deformações para estudo do efeito Payne em nanocompósitos não vulcanizados de NBR/MMTorg:

É possível observar pelos resultados da Tabela 3 que a diferença do módulo elástico $\left(\Delta \mathrm{G}^{\prime}=\mathrm{G}^{\prime} 14 \%-\mathrm{G}^{\prime} 210 \%\right)$ aumenta com a adição crescente de carga, indicando o rompimento de maior número de interações carga-carga. Estas interações são consequência da formação de aglomerados de MMTorg presente na matriz de NBR.

Com base na literatura ${ }^{[25-33]}$, grandes teores de aglomerados na matriz elastomérica causam prejuízo às propriedades de uma forma geral, e as propriedades dinâmicas em particular.

Os resultados obtidos favorecem a composição com $5 \mathrm{phr}$ de MMTorg significando melhor interação polímero-carga como consequência da menor quantidade de aglomerados na matriz elastomérica, corroborando os estudos por difração de raios- $\mathrm{X}^{[33]}$.

\section{Conclusões}

O processamento usado neste trabalho para a incorporação de MMTorg em NBR foi eficiente na obtenção de nanocompósitos, que foram caracterizados e comprovados por microscopia eletrônica de transmissão.

A análise de difração de raios-X permitiu diferenciar as características da MMTorg na matriz de borracha nitrílica (NBR), indicando o teor limite na formação dos nanocompósitos.

O analisador de processamento de borracha - RPA 2000 permitiu estudar através do Efeito Payne as interações carga-carga e polímero carga, usando uma ampla faixa de deformação em função do módulo elástico das composições, que foram utilizadas em aplicações dinâmicas.

Os resultados mostraram que a composição com $5 \mathrm{phr}$ de MMTorg apresentou melhor interação polímero-carga como consequência da menor quantidade de aglomerados de carga na matriz elastomérica.

\section{Agradecimentos}

Os autores agradecem aos órgãos de fomento CNPq, CAPES, FAPERJ e FUJB pelo apoio financeiro e a Nitriflex S.A. Indústria e Comércio pelo fornecimento da borracha nitrílica e dos aditivos usados neste trabalho.

\section{Referências Bibliográficas}

1. Wang, S.; Long, C.; Wang, X.; Li, Q. \& Qi, Z. - J. Appl. Polym Sci., 69, p.1557 (1998). http://dx.doi.org/10.1002/(SICI) 10974628(19980822)69:8<1557::AID-APP10>3.0.CO;2-S

2. Przybyszewska, M. \& Zaborski, M. - Polym. Lett., 3, p.542 (2009). http://dx.doi.org/10.3144/expresspolymlett.2009.68

3. Wu, Y. P.; Jia, Q. X.; Yu, D. S. \& Zhang, L. Q. - Polym. Test., 23, p.903 (2004). http://dx.doi.org/10.1016/j.polymertesting.2004.05.004

4. Kim, J. T.; Oh, T. S. \& Lee, D. H. - Polym. Int., 52, p.1058 (2003). http://dx.doi.org/10.1002/pi.1110
5. Hwang, W. G.; Wei, K. H. \& Wu, C. M. - Polymer, 45, p.5729 (2004). http://dx.doi.org/10.1016/j.polymer.2004.05.040

6. Alexandre, M. \& Dubois, P. - Mat. Sci. Eng.; 28, p.1 (2000). http:// dx.doi.org/10.1016/S0927-796X(00)00012-7

7. Kim, J. T.; Oh, T. S. \& Lee, D. H. - Polym. Int., 52, p.1203 (2003). http://dx.doi.org/10.1002/pi.1249

8. Mitra, S.; Chattopadhyay, S. \& Bhowmick, A. K. - J. Appl. Polym. Sci., 118, p.81 (2010).

9. Balachandran, M.; Stanly, L. P.; Mulaleekrishnan, R. \& Bhagawan, S. S. - J. Appl. Polym. Sci., 118, p.3300 (2010). http://dx.doi.org/10.1002/ app. 32147

10. Paiva, L. B.; Morales, A. R. \& Díaz, F. R. V. - Cerâmica, 54, p.213 (2008).

11. Bezerra, F. O. - "Desenvolvimento de Nanocompósitos de NBR com Montmorilonita Organofílica”, Dissertação de Mestrado, Universidade Federal do Rio de Janeiro, Brasil (2007).

12. Janowska, G.; Kucharska-Jastrza, A. \& Rybinski, P. - J. Therm. Anal. Calorim., 103, p.1039 (2011). http://dx.doi.org/10.1007/s10973-0101282-y

13. Barbosa, R.; Souza, D. D.; Araújo, E. M. \& Mélo, T. J. A. - Cerâmica, 56, p.376 (2010).

14. Hwang, W. G. \& Wei, K. H. - Polym. Eng. Sci., 44, p.2117 (2004). http://dx.doi.org/10.1002/pen.20217

15. Han, M.; Kim, H. \& Kim, E. - Nanotechnology, 17, p.403 (2006). http://dx.doi.org/10.1088/0957-4484/17/2/010

16. Wu, Y. P.; Wang, Y. Q.; Zhang, H. F.; Wang,Y. Z.; Yu, D. S.; Zhang, L. Q. \& Yang, J. - Comp. Sci. Techn., 65, p.1195 (2005). http://dx.doi. org/10.1016/j.compscitech.2004.11.016

17. Lepoittevin, B.; Pantoustier, N.; Devalckenaere, M.; Alexandre, M.; Calberg, C.; Jérôme, R.; Henrist, C.; Rulmont, A. \& Dubois, P. - Polymer, 44, p.2033 (2003). http://dx.doi.org/10.1016/S00323861(03)00076-4

18. Gerspacher, M.; O'Farrell, C. P.; Yang, H. H. \& Nikiel, L. - Rubber World, April, p.27 (1999).

19. Gerspacher, M. \& O'Farrell, C. P. - Kaut. Gummi. Kunstst, 51, p.488 (1998).

20. Bandyopadhyay, S.; De, P. P.; Tripathy, D. K. \& De, S. K. - Polymer, 37, p.353 (1996). http://dx.doi.org/10.1016/0032-3861(96)81110-4

21. Tehovnik, M. D.; Marinovic, T. \& Susteric, Z. - Kaut. Gummi. Kunstst, 50, p.613 (1997).

22. Kader, M. A.; Kim, K.; Lee, Y. S. \& Nah, C. - J. Mater. Sci., 41, p.7341 (2006). http://dx.doi.org/10.1007/s10853-006-0792-2

23. Kornmann, X.; Lindeberg, H. \& Berglund, L. A. - Polymer, 42, p.1303 (2001). http://dx.doi.org/10.1016/S0032-3861(00)00346-3

24. Wang, M. J. - Rubber Chem. Technol., 71, p.520 (1998). http://dx.doi. org/10.5254/1.3538492

25. Maier, P. G. \& Göritz, D. R. - Kaut. Gummi. Kunstst, 49, p.18 (1996).

26. Donnet, J. B. - Compos. Sci. Technol., 63, p.1085 (2003). http://dx.doi. org/10.1016/S0266-3538(03)00028-9

27. Leblanc, J. L. - Prog. Polym. Sci., 27, p.627 (2002). http://dx.doi. org/10.1016/S0079-6700(01)00040-5

28. Lapa, V. L. C. - "Efeito Payne em elastômeros", Seminário para Doutorado, Instituto de Macromoléculas Professora Eloisa Mano - Universidade Federal do Rio de Janeiro, Brasil (2004).

29. Payne, A. R. - J. Appl. Polym. Sci., 19, p.57 (1962). http://dx.doi. org/10.1002/app.1962.070061906

30. Payne, A. R. \& Whittaker, R.E. - Rubber Chem. Technol., 44, p.440 (1971). http://dx.doi.org/10.5254/1.3547375

31. Niedermeier, W.; Luginsland, J. F. \& Köln, H.-D. - Kaut. Gummi. Kunstst., 7-8, p.356 (2002). 
32. Medalia, I.A. - Rubber Chem. Technol., 51, p.437 (1978). http://dx.doi. org/10.5254/1.3535748

33. Cassagnau, P. - Polymer, 44, p.2455 (2003). http://dx.doi.org/10.1016/ S0032-3861(03)00094-6

34. Das, A.; Jurk, R.; Stöckelhuber, K. W. \& Heinrich, G. - Polym. Lett., 1, p.717(2007). http://dx.doi.org/10.3144/expresspolymlett.2007.99

35. Hubery, G.; Vilgisy, T. A. \& Heinrichz, G. - J. Phys.: Condens. Matter 8 , L409 (1996). http://dx.doi.org/10.1088/0953-8984/8/29/003

36. Gauthier, C.; Reynaud, E.; Vassoillea, R. \& Ladouce-Stelandre, L. - Polymer 45, p.2761 (2004). http://dx.doi.org/10.1016/j. polymer.2003.12.081

37. Dick, J. S. \& Pawlowski, H. A. - "Applications for the Rubber Process Analyser", in: Proceedings of the Rubber Division, American Chemical Society, p.1, Nashville, nov. (1993).
38. Dick, J. S. \& Pawlowski, H. A. - "Rubber Characterization by Applied Strain Variations using the Rubber Process Analyzer", in: Proceedings of the Rubber Division, American Chemical Society, p.1-21, Chicago, Illinois, April (1994).

39. Pawlowski, H. A. \& Barker, R. I. - "Measurement of the Viscoelastic Properties of Elastomers with a new Dynamic Mechanical Rheological Tester", in: Proceedings of the Plastics \& Rubber Institute, Leuven, Belgium, April 19-20 (1994).

40. Soares, B. G.; De Oliveira, M. \& Zaioncz, S. - Polímeros, 20, p.371 (2010). http://dx.doi.org/10.1590/S0104-14282010005000055

41. Drozdov, A. D. \& Dorfmann, A. - Polym. Eng. Sci., 42, p.591 (2002). http://dx.doi.org/10.1002/pen.10974

42. Frohlich, J.; Niedermeier, W. \& Luginsland, H.-D. - Compos.: Part A, 36, p.449 (2005).

43. Baumhard Neto, R. - "Raios X”, in: Técnicas de Caracterização de Polímeros, S.V. Canevarolo, Artliber Editora Ltda, São Paulo (2007).

Enviado: $31 / 08 / 11$

Reenviado: 02/03/12

Aceito: $14 / 04 / 12$ 